CLINICAL STUDY

\title{
Association study of the polymorphisms in the KISS1 gene with central precocious puberty in Chinese girls
}

\author{
Xiaohui Luan ${ }^{1,2}$, Yuxun Zhou ${ }^{2}$, Wei Wang ${ }^{3}$, Hong $\mathrm{Yu}^{4}$, Pin $\mathrm{Li}^{5}$, Xiaohong Gan $^{2}$, Dongzhi Wei ${ }^{1}$ and Junhua Xiao ${ }^{2,1}$ \\ ${ }^{1}$ State Key Laboratory of Bioreactor Engineering, Institute of Biochemistry, East China University of Science and Technology, 130 Meilong Road, Shanghai \\ 200237, People's Republic of China, ${ }^{2}$ Institute of Bioscience and Technology, Donghua University, 1882 West Yan'an Road, Shanghai 200052, People's \\ Republic of China, ${ }^{3}$ Department of Pediatrics, Ruijin Hospital, Shanghai Jiaotong University, 197 No. 2 Ruijin Road, Shanghai 200025, People's Republic \\ of China, ${ }^{4}$ Department of Adolescent Clinic, Harbin Children's Hospital, 57 Youyi Road, Harbin 150010, People's Republic of China and ${ }^{5}$ Department of \\ Endocrinology, Shanghai Jiaotong University Affiliated Children's Hospital, 1400 West Beijing Road, Shanghai 200040, People's Republic of China \\ (Correspondence should be addressed to J Xiao; Email: xiaojunhua@dhu.edu.cn)
}

\begin{abstract}
Objective: The kisspeptin/GPR54 pathway has been proven to be crucial in the process of puberty onset, yet the polymorphisms in the KISS1 gene and their relationships with central precocious puberty (CPP) have not been investigated. This study was performed to reveal the relationship between the gene and the disease.

Design and Methods: 272 Chinese Han girls diagnosed to be CPP patients were recruited as Case Group I, 43 unrelated African women as Case Group II, and 288 unrelated normal Chinese Han girls as Control Group. Polymorphism scans of the KISS1 gene were performed for the first time by bidirectional resequencing of the whole gene in a subset of the patients, and then by ligase detection reaction some of the polymorphisms identified were typed in the two groups and the respective haplotypes were constructed. The relationships of the typed polymorphisms and the haplotypes with CPP were evaluated by an association study between genotypes and phenotypes.

Results: By resequencing, eight polymorphisms were identified, five of which were typed forming 18 haplotypes. Although one novel nonsynonymous single nucleotide polymorphism substituting one amino acid in kisspeptin (P110T) was found to be statistically related to the disease $(P=0.025)$, no further supporting evidence has yet been found. The other polymorphisms and all the haplotypes were not found to be related.

Conclusion: The polymorphism scanning and typing of KISS1 uncovered several potentially meaningful polymorphisms, but the conclusion was not solid and further studies are necessary for function validation of these polymorphisms.
\end{abstract}

European Journal of Endocrinology 157 113-118

\section{Introduction}

When kisspeptin was first identified in 1996 (1), it was mainly considered as a tumor metastasis suppressor. Since it was found to be the ligand of orphan G-protein coupled receptor GPR54 in 2001 (2), researchers came to notice the possible role of kisspeptin/GPR54 signal pathway in the regulation of endocrine function, which was proved by recent studies to demonstrate that kisspeptin would directly activate the onset of puberty in mammals via GPR 54 by stimulating the secretion of gonadotropin-releasing hormone (GnRH; (3-5)).

The onset timing of puberty varies depending on numerous variables. Generally, it would be defined as precocious puberty if any signs of secondary sexual maturation appears at an age more than 2 standard deviation units below the population mean $(<8$ for girls and $<9$ for boys in China), and would be further defined as central precocious puberty (CPP) if the process is driven by activation of hypothalamic GnRH secretion.
There have been reports indicating that CPP patients would be shorter in stature when fully grown and more susceptible to breast cancer (6). The incidence of CPP in Chinese children was about $1 \%$ according to epidemiologic data in 2003 (7). Some studies have identified several associated genes or polymorphisms involved in development, body composition, gonadal steroid metabolism, and many other biological processes $(8-10)$, yet the exact mechanism remains a mystery. The important function of kisspeptin in puberty onset makes it necessary to investigate the polymorphisms in the gene and their relationships with CPP.

The KISS1 gene consists of three exons, of which only part of the second and third exons are finally translated into a 145-amino acid protein with a putative signal sequence, from which three forms of kisspeptins containing 54, 14, and 13 amino acids are derived (Fig. 1). The three peptides exhibit the same affinity for the receptor since they share a common C-terminal 
KISS1 Protein (145 AA)

MNSLVSWQLLLFLCATHFGEPLEKVASVGNSRPTGQQLESLGLLAPGEQSLPCTERKPAAT ARLSRRGTSLSPPPESSGSPQQPGLSAPHSRQIPAPQGAVLVQREKDLPNYNWNSFGLRF GKREAAPGNHGRSAGRGWGAGAGQ

Kisspeptin-54

GTSLSPPPESSGSPQQPGLSAPHSRQIPAPQGAVLVQREKDLPNYNWNSFGLRF-NH ${ }_{2}$

Kisspeptin-14
DLPNYNWNSFGLRF-NH ${ }_{2}$

Kisspeptin-10

YNWNSFGLRF-NH
Kisspeptin-13

LPNYNWNSFGLRF-NH
Figure 1 Amino acid sequence of the KISS1 product and derived peptides. The predicted signal peptide of KISS1 is in italicized and bold letters. Dibasic motifs representing potential cleavage sites by furins and prohormone convertases are underlined and in bold letters. The two amino acids changed by $54650055 \mathrm{G} / T$ and $54650141 \mathrm{G} / \mathrm{C}$ are marked within rectangles. decapeptide designated kisspeptin-10, which is the minimum sequence necessary for receptor activation.

In this study, the three exons, exon-intron boundaries, and $1.4 \mathrm{~kb}$ of $5^{\prime}$ flanking region of the KISS1 gene were amplified and sequenced in a cohort of 36 Chinese CPP girls, by which the mechanism of how how the kisspeptin/GPR54 system functions in the onset of puberty may be better understood.

\section{Material and methods}

\section{Experimental subjects}

Three groups of subjects were involved in this study. Case Group I consisted of 272 Chinese sporadic CPP girls recruited from three hospitals in Shanghai and Harbin, China; Case Group II consisted of 43 normal African adult female subjects; and Control Group consisted of 288 independent normal Chinese Han girls recruited from East China University of Science and Technology. The study protocols were reviewed and approved by the Ethics Committees for Human Research of the hospitals and the college, and informed consent was obtained from all the subjects or their guardians.

The CPP patients were all diagnosed according to the same criteria and at diagnosis they were between 5.9 and 8.5 years of age. The patients all showed dramatic growth acceleration, with Tanner breast stage scores $\geq 2$ based on standardized Tanner breast stage assessment. Bone ages were measured and data of 215 subjects $(79.04 \%)$ were found to be at least 1 year ahead of their true ages, and a few of the patients even had vaginal bleeding. A GnRH stimulation test was carried out in all subjects to confirm that the hypothalamic-pituitary-gonadal axis had been activated, by which significantly high luteinizing hormone $(\mathrm{LH})$ peak values $(23.2 \pm 12.7 \mathrm{mIU} / \mathrm{ml})$ were observed after $40 \mathrm{~min}$ of the injection, when compared with the cut-off value of $15 \mathrm{mIU} / \mathrm{ml}$ (11), and the LH/follicle-stimulating hormone (FSH) ratios were all above 0.66 (12). To exclude any possible brain abnormalities, magnetic resonance imaging was performed and no brain diseases were found. Most of the parents of the patients were questioned about their pubertal ages, yet none of them recalled precocious puberty in childhood.

The DNA samples of the African subjects were obtained from the Chinese National Human Genome Center at Shanghai. The 43 subjects were all normal African women with a mean age of $36.8 \pm 12.5$ years. The puberty onset timing of the subjects was not obtained.

The control subjects were selected from female volunteers who were first grade students in East China University of Science and Technology based on a freewill questionnaire regarding their age of menarche. The mean age of the control subjects was $18.24 \pm 1.38$ and the mean age of menarche was above 12 years.

In total, $36 \mathrm{CPP}$ subjects were randomly selected from Case Group I for sequencing.

Peripheral blood samples were collected from all subjects and genome DNA samples were isolated with phenol-chloroform method.

\section{Sequencing}

Six pairs of overlapping primers covering all the sequenced regions were designed and synthesized (Sangon, Shanghai, China), with which PCR was performed in a $20 \mu \mathrm{l}$ system by 35 cycles of amplification. The products were purified for bidirectional sequencing on an ABI 3100 sequencer (Applied Biosystems, Foster City, CA, USA).

\section{Genotyping}

Typing of the selected single nucleotide polymorphisms (SNPs) was performed with a method developed by 
ligase detection reaction (LDR; (13)), the mechanism of which is shown in Fig. 2. Multiplex PCR was performed to amplify four fragments containing the five SNPs with HotStarTaq polymerase (Qiagen Inc.) in a volume of $20 \mu \mathrm{l}$ by 40 cycles of amplification.

The PCR products were used as templates for LDR. For each polymorphism, one universal fluorescencelabeled probe and two allele-specific probes of different lengths were designed according to the flanking sequence. The LDR was carried out in a volume of $20 \mu \mathrm{l}$ containing probes, PCR products and thermostable Taq DNA ligase (New England Biolabs, Ipswich, MA, USA) according to the instructions. Recognizing respective alleles, the probes would be joined by ligase into corresponding products distinguishable on ABI 3100 sequencer. The electrophoresis results were analyzed by GeneMapper ID software version 3.2 (Applied Biosystems). Primers and probes were also synthesized by Sangon, and oligo sequences and detailed reaction conditions can be supplied on request.
Deviations from the Hardy-Weinberg equilibrium were tested by comparison of observed and expected genotype frequencies with $\chi^{2}$ test.

\section{Haplotype construction and statistical analysis}

Haplotypes were estimated from genotype data for individual participants using the PHASE program version 2.0 (14).

The comparison of the allele frequencies among the three groups was performed by Fisher's exact test. The SPSS 11.5 software package (SPSS Inc., Chicago, IL, USA) was used to perform statistical analysis with $P<$ 0.05 as the minimal level of statistical significance.

\section{Results}

Sequencing of the KISS1 gene revealed eight SNPs as listed in Table 1, among which four were identified for
$54650055 \mathrm{G}$

Homozygous

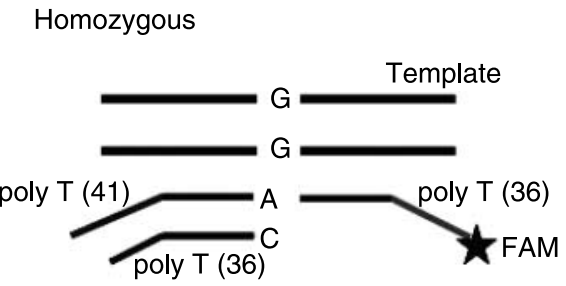

Allele-specific probes Universal probe
$54650141 \mathrm{G} / \mathrm{C}$

Heterozygous
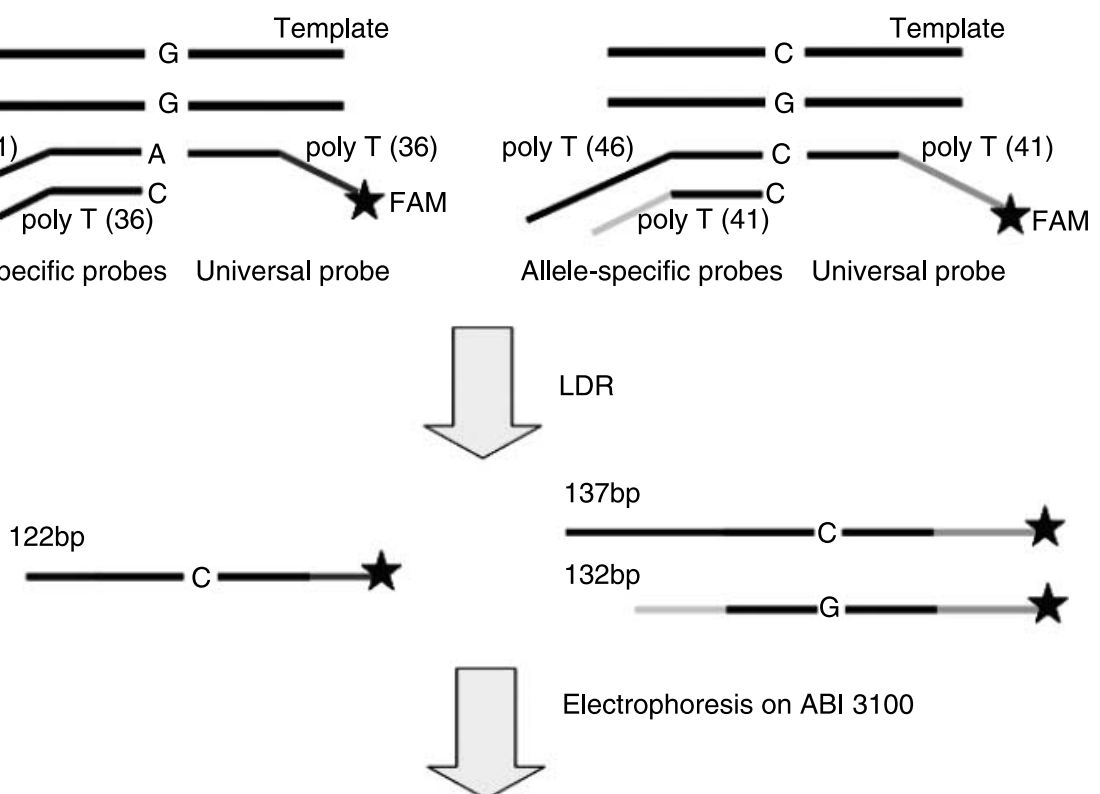

Electrophoresis on ABI 3100

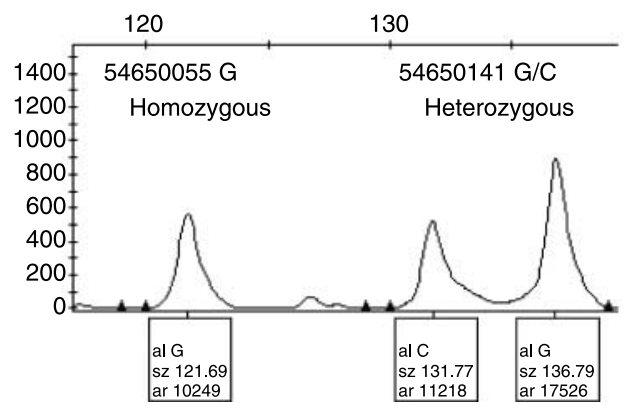

Figure 2 Flow chart of genotyping method based on ligase detection reaction. A sample which was wild-type homozygous at 54650055 and heterozygous at 54650141 was selected to explain the mechanism of the genotyping method. The trace map at the bottom was generated with the software GeneMapper ID version 3.2. 
Table 1 Single nucleotide polymorphisms (SNPs) identified by sequencing.

\begin{tabular}{|c|c|c|c|c|c|c|c|}
\hline No. & Position $^{a}$ & Allele & Location & dbSNP ID & $\begin{array}{l}\text { Frequency in } \\
\text { sequenced } \\
\text { samples }\end{array}$ & Typed & Note \\
\hline 1 & 54656335 & $A / G$ & $5^{\prime}$ flanking region & 1 & $0.897 / 0.103$ & Yes & \\
\hline 2 & 54656397 & $C / T$ & $5^{\prime}$ flanking region & rs3924 586 & $0.651 / 0.349$ & Yes & \\
\hline 3 & 54656436 & $G / A$ & $5^{\prime}$ flanking region & rs3924 585 & $0.939 / 0.061$ & No & \\
\hline 4 & 54656861 & $A / T$ & $5^{\prime}$ flanking region & 1 & $0.983 / 0.017$ & No & \\
\hline 5 & 54655907 & $C / T$ & Exon 1 & 1 & $0.944 / 0.056$ & No & Untranslated \\
\hline 6 & 54655965 & $A /-$ & Exon 1 & rs5780 218 & $0.5 / 0.5$ & Yes & Untranslated \\
\hline 7 & 54650055 & $G / T$ & Exon 3 & / & $0.943 / 0.057$ & Yes & AA change: P110T \\
\hline 8 & 54650141 & $G / C$ & Exon 3 & rs4889 & $0.542 / 0.458$ & Yes & AA change: P81R \\
\hline
\end{tabular}

${ }^{\text {a }}$ The positions of the SNPs were defined according to contig NT_004487.18.

the first time (sequencing traces shown in Fig. 3). Two SNPs $(54650055 \mathrm{G} / \mathrm{T}$ and $54650141 \mathrm{G} / \mathrm{C})$ were found to be nonsynonymous, leading to substitution of P110T and P81R respectively.

Five SNPs of relatively high heterozygosity were selected and genotyped in the three groups, and the results fitted the expectation given by the HardyWeinberg equilibrium. Allele counts and frequencies are shown in Table 2a. With Fisher's exact test, the relationships between the polymorphisms and the phenotypes were evaluated, among which the nonsynonymous polymorphism $54650055 \mathrm{G} / \mathrm{T}$ showed statistical significance with CPP $(P=0.025)$, while the other four SNPs showed none.

In total 18 haplotypes were constructed in Case Group I and Control Group based on the typing data, and their relationships with CPP were also investigated (Table $2 b$ ), yet none of them showed significant correlation. a

54656335 A Homozygous

80

b

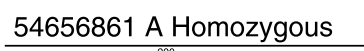

\section{Discussion}

GPR54 remained an orphan receptor until three independent groups $(2,15,16)$ identified its natural ligand kisspeptin. Numerous studies on various species including mice, rats, sheep, primates and humans had reported that the kisspeptin/GPR54 pathway would stimulate the secretion of GnRH and start the onset of puberty $(3,17-19)$. Several mutations were found in the GPR 54 gene and their functions demonstrated, yet no such studies had been carried out on the KISS1 gene. This study was the first time that the polymorphisms in KISS1 and their relationships with CPP were investigated in Chinese girls.

Whole gene resequencing was carried out in part of the CPP patients to screen polymorphisms, after which the frequencies of the eight SNPs found in the sequencing were calculated in the sequenced cohort, and five were considered informative due to their

C

54655907 C Homozygous G A G O T G A $\stackrel{90}{C}$ G A G A O C A

d

54650055 G Homozygous
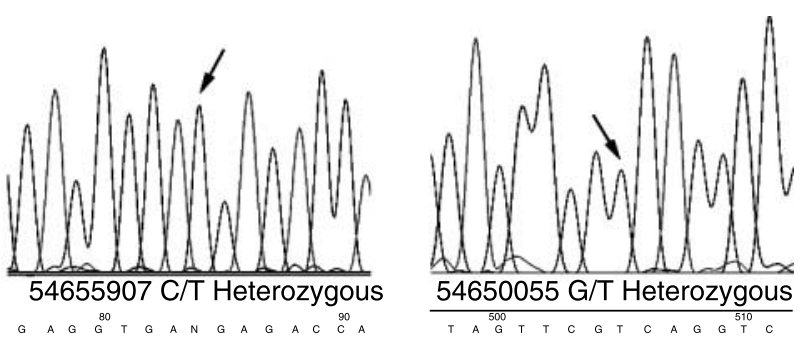

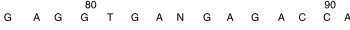

T A Q T T T C G G C A G G T C
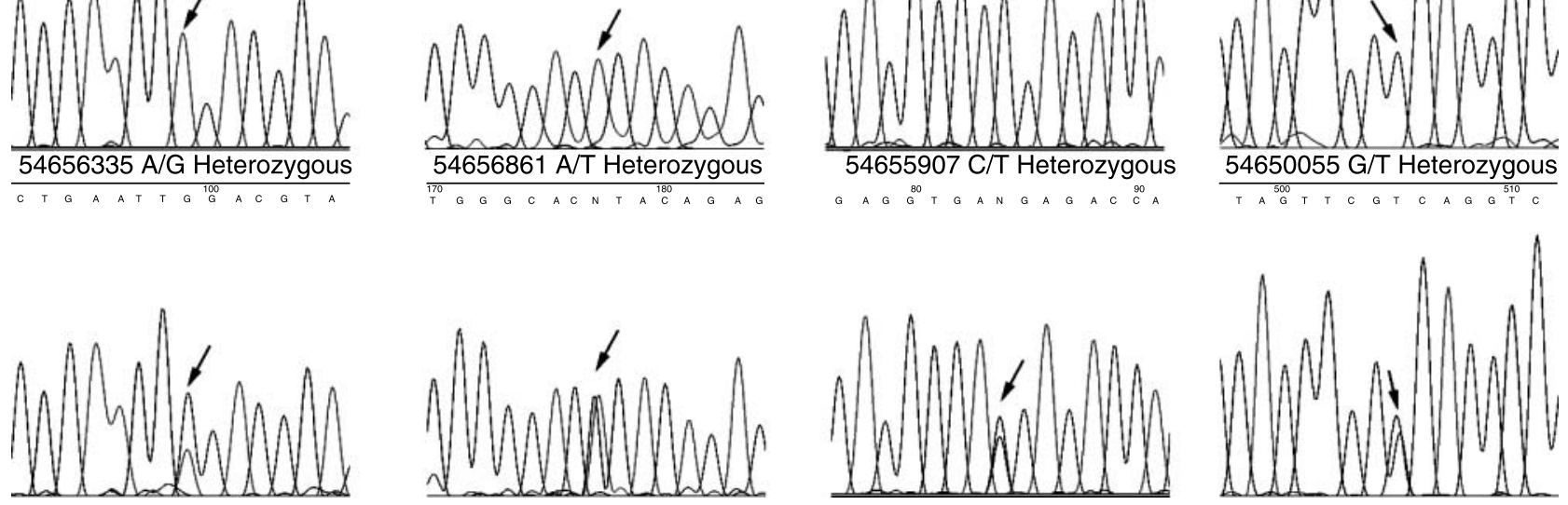

Figure 3 Sequencing results of novel polymorphisms. Typical sequencing traces of the four novel single nucleotide polymorphism are shown, wild-type homozygous subjects on the top and heterozygous genotype on the bottom. The arrows indicate the positions of polymorphisms. 
Table 2 Genotyping results and haplotype analysis.

\begin{tabular}{|c|c|c|c|c|c|c|c|c|c|c|c|c|c|c|}
\hline \multirow{2}{*}{\multicolumn{2}{|c|}{ SNP }} & \multirow[b]{2}{*}{ Group } & \multicolumn{4}{|c|}{ Allele counts (frequencies) } & \multicolumn{6}{|c|}{ Genotype counts (frequencies) } & \multicolumn{2}{|c|}{$P$-value } \\
\hline & & & & 1 & & 2 & & 1 & & 2 & & 22 & $\begin{array}{l}\text { Case I- } \\
\text { Control }\end{array}$ & $\begin{array}{c}\text { Case II - } \\
\text { Control }\end{array}$ \\
\hline \multicolumn{15}{|c|}{ (a) Genotyping results } \\
\hline \multirow{3}{*}{\multicolumn{2}{|c|}{$54656335 A / G A=1, G=2$}} & Case I & 519 & 0.954 & 25 & 0.046 & 247 & 0.908 & 25 & 0.092 & 0 & 0 & 0.660 & 0.251 \\
\hline & & Case II & 80 & 0.930 & 6 & 0.070 & 37 & 0.860 & 6 & 0.140 & 0 & 0 & & \\
\hline & & Control & 553 & 0.960 & 23 & 0.040 & 265 & 0.920 & 23 & 0.080 & 0 & 0 & & \\
\hline \multirow{3}{*}{\multicolumn{2}{|c|}{$54656397 C / T C=1 ; T=2$}} & Case I & 379 & 0.697 & 165 & 0.303 & 138 & 0.507 & 103 & 0.379 & 31 & 0.114 & 0.209 & 0.796 \\
\hline & & Case II & 61 & 0.709 & 25 & 0.291 & 22 & 0.512 & 17 & 0.395 & 4 & 0.093 & & \\
\hline & & Control & 421 & 0.731 & 155 & 0.269 & 152 & 0.528 & 117 & 0.406 & 19 & 0.066 & & \\
\hline \multirow{3}{*}{\multicolumn{2}{|c|}{$54655965 A /-A=1, \mathrm{Del}=2$}} & Case I & 261 & 0.480 & 283 & 0.520 & 57 & 0.210 & 147 & 0.540 & 68 & 0.250 & 0.187 & 0.417 \\
\hline & & Case II & 42 & 0.488 & 44 & 0.512 & 9 & 0.209 & 24 & 0.558 & 10 & 0.233 & & \\
\hline & & Control & 253 & 0.439 & 323 & 0.561 & 57 & 0.198 & 139 & 0.483 & 92 & 0.319 & & \\
\hline \multirow{3}{*}{\multicolumn{2}{|c|}{$54650055 G / T G=1, T=2$}} & Case I & 523 & 0.961 & 21 & 0.039 & 251 & 0.923 & 21 & 0.077 & 0 & 0 & 0.025 & 0.006 \\
\hline & & Case II & 86 & 1 & 0 & 0 & 43 & 1 & 0 & 0 & 0 & 0 & & \\
\hline & & Control & 536 & 0.931 & 40 & 0.069 & 248 & 0.861 & 40 & 0.139 & 0 & 0 & & \\
\hline \multirow{3}{*}{\multicolumn{2}{|c|}{$54650141 G / C G=1 ; C=2$}} & Case I & 316 & 0.581 & 228 & 0.419 & 91 & 0.335 & 134 & 0.493 & 47 & 0.173 & 0.856 & 0.640 \\
\hline & & Case II & 48 & 0.558 & 38 & 0.442 & 13 & 0.302 & 22 & 0.512 & 8 & 0.186 & & \\
\hline & & Control & 338 & 0.587 & 238 & 0.413 & 101 & 0.351 & 136 & 0.472 & 51 & 0.177 & & \\
\hline & \multicolumn{4}{|c|}{ Case I } & \multicolumn{6}{|c|}{ Control } & \multirow{2}{*}{\multicolumn{2}{|c|}{ Total }} & \multirow{2}{*}{\multicolumn{2}{|c|}{$P$-value }} \\
\hline Haplotype & Count & & Frequ & iency & & Count & & Freq & uency & & & & & \\
\hline \multicolumn{15}{|c|}{ (b) Haplotype analysis } \\
\hline AC-GC & 172 & & \multirow{2}{*}{\multicolumn{2}{|c|}{$\begin{array}{l}0.3162 \\
0.1048\end{array}$}} & \multicolumn{3}{|c|}{178} & \multicolumn{2}{|c|}{0.3090} & & \multicolumn{2}{|c|}{350} & \multicolumn{2}{|c|}{0.797} \\
\hline$A C-G G$ & 57 & & & & & 81 & & & 1406 & & 13 & & & .070 \\
\hline AC-TC & 1 & & \multicolumn{2}{|c|}{0.0018} & & 1 & & & 017 & & & 2 & & .000 \\
\hline AC-TG & 2 & & 0.0 & 37 & & 7 & & & 122 & & & 9 & & .180 \\
\hline ACAGC & 9 & & 0.0 & 165 & & 18 & & & 313 & & 2 & 7 & & .122 \\
\hline ACAGG & 111 & & 0.2 & 40 & & 103 & & & 1788 & & 21 & & & .288 \\
\hline ACATC & 0 & & 0 & & & 4 & & & 0069 & & & 4 & & .125 \\
\hline ACATG & 17 & & 0.0 & 313 & & 24 & & & 417 & & 4 & 1 & & .427 \\
\hline AT-GC & 20 & & 0.0 & 368 & & 17 & & & 295 & & 3 & 7 & & .509 \\
\hline AT-GG & 11 & & 0.0 & 202 & & 20 & & & 347 & & 3 & 1 & & 149 \\
\hline ATAGC & 24 & & 0.0 & 441 & & 16 & & & 278 & & 4 & 0 & & .150 \\
\hline ATAGG & 95 & & 0.1 & 746 & & 84 & & 0.1 & 1458 & & 17 & & & .193 \\
\hline $\mathrm{GC}-\mathrm{GC}$ & 1 & & 0.0 & 18 & & 0 & & 0 & & & & 1 & & .486 \\
\hline GC-GG & 4 & & 0.0 & 074 & & 1 & & & 0017 & & & 5 & & .205 \\
\hline GCAGG & 4 & & 0.0 & 074 & & 0 & & 0 & & & & 4 & & .055 \\
\hline GCATC & 1 & & 0.0 & 18 & & 4 & & & 069 & & & 5 & & .375 \\
\hline GT-GC & 0 & & 0 & & & 1 & & & 0017 & & & 1 & & .000 \\
\hline GT-GG & 15 & & 0.0 & 276 & & 17 & & & 295 & & 3 & 2 & & .860 \\
\hline
\end{tabular}

relatively high degree of heterozygosity and were selected as 'tagging' SNPs for genotyping. Though the frequency of $54656436 \mathrm{G} / \mathrm{A}$ was a little higher than that of $54650055 \mathrm{G} / \mathrm{T}$ in the sequenced cohort, the latter was selected for typing since it was an amino acid substituting polymorphism.

According to the statistical results calculated with the typing data, the nonsynonymous SNP 54650055 G/T substituting the 110th amino acid of KISS1 protein from proline to threonine was found to be statistically related to CPP. Though the P110T site was contained by all the three natural in vivo forms of kisspeptin, kisspeptin-54, 14, and 13 (Fig. 1), the association could not be validated without further support from functional data, especially considering that the sample scale was relatively small and that a $P$-value of 0.025 was not significant enough.
A different ethnic group was involved for investigation to provide more solid evidence. From the aspect of human evolution and migration, delayed puberty onset would be observed as humans moved to colder regions, as described in a recent review (20) which showed that African-American children had earlier pubertal milestones than white children, with the data of MexicanAmerican children in between. The facts can be explained by the assumption that individuals tend to delay the onset of puberty when facing tougher environments - less food and lower temperature. Fortythree healthy African female adults were then selected as Case Group II despite the lack of their pubertal data. The five tagging SNPs were also typed with the same method in all the subjects, and the results (also shown in Table 2) showed that four SNPs were of similar frequencies as observed in Chinese subjects, except $54650055 \mathrm{G} / \mathrm{T}$, 
which was not found in any of the African subjects. The $P$-value between Case Group II and Control Group was 0.006. Based on the data, it may be reasonable to hypothesize that the mutation could in some degree protect the carrier from earlier puberty onset.

The other nonsynonymous SNP did not exhibit significant relationship either between Case Group I and Control Group, or between Case Group II and Control Group. The polymorphism introduced a substitution at the 81 st site from proline to arginine, which was included by kisspeptin- 54 but not by the other three forms. From the result, it may be assumed that kisspeptin-14 and 13 are possibly more important than kisspeptin-54 in humans; thus, the amino acid change would not seriously affect the bioactivity, supported by a previous report that kisspeptin-54 was unstable and would easily degrade into the other two forms (2).

The association study of haplotypes constructed with the five SNPs typed showed no significant relationship between the 18 haplotypes, which could be partially attributed to the relatively small scale of population typed, causing the correlated alleles to disperse into different haplotypes. Owing to this, it was more difficult to confirm the relationship between the haplotypes and the disease.

In brief, this study tried to reveal the relationships between the polymorphisms and haplotypes in KISS1 gene and CPP in Chinese girls. The results would deepen our understanding in both the KISS1 gene and the mechanism of the disease. Yet, the limit of association study calls for more research in the future to confirm the actual function of the polymorphism.

\section{Acknowledgements}

This work was supported by grants from the National Nature Science Foundation of China (Grant no. 30300 198) and the State Key Basic Research Program (Grant no. 2002CB512 900).

\section{References}

1 Lee J-H, Miele ME, Hicks DJ, Phillips KK, Trent JM, Weissman BE \& Welch DR. KiSS-1, a novel human malignant melanoma metastasis- suppressor gene. Journal of the National Cancer Institute 199688 1731-1737.

2 Kotani M, Detheux M, Vandenbogaerde A, Communi D, Vanderwinden JM, Poul EL, Brezillon S, Tyldesley R, SuarezHuerta N, Vandeput F, Blanpain C, Schiffmann SN, Vassart G \& Parmentier M. The metastasis suppressor gene KiSS-1 encodes kisspeptins, the natural ligands of the Orphan G Protein-coupled receptor GPR54. Journal of Biological Chemistry 2001276 34631-34636.

3 Messager S, Chatzidaki EE, Ma D, Hendrick AG, Zahn D, Dixon J, Thresher RR, Malinge I, Lomet D, Carlton MBL, Colledge WH, Caraty A \& Aparicio S. Kisspeptin directly stimulates gonadotropin-releasing hormone release via $\mathrm{G}$ protein-coupled receptor 54. PNAS $20051021761-1766$.
4 Irwig MS, Fraley GS, Smith JT, Acohido BV, Popa SM, Cunningham MJ, Gottsch ML, Clifton DK \& Steiner RA. Kisspeptin activation of gonadotropin releasing hormone neurons and regulation of KiSS-1 mRNA in the male rat. Neuroendocrinology $200480264-272$.

5 Dhillo WS, Chaudhri OB, Patterson M, Thompson EL, Murphy KG, Badman MK, McGowan BM, Amber V, Patel S, Ghatei MA \& Bloom SR. Kisspeptin-54 stimulates the hypothalamic-pituitary gonadal axis in human males. Journal of Clinical Endocrinology and Metabolism 200590 6609-6615.

6 Petridou E, Syrigou E, Toupadaki N, Zavitsanos X, Willett W \& Trichopoulos D. Determinants of age at menarche as early life predictors of breast cancer risk. International Journal of Cancer 1996 68 193-198.

7 Cai DP. Precocious and Delayed Puberty in Children. 1 Shanghai: Fudan University Press, 2003 p 35.

$8 \mathrm{Yu} \mathrm{WH}$, Kimura M \& Walczewaka A. Role of leptin in hypothalamic-pituitary function. PNAS 199794 1023-1028.

9 Kadlubar FF, Berkowitz SG, Delongchamp RR, Charles W, Green LB, George T, Jatinder L, Erin S \& Mary SW. The CYP $3 A 4^{*} 1 B$ variant is related to the onset of puberty, a known risk factor for the development of breast cancer. Cancer Epidemiology, Biomarkers and Prevention 200512 327-331.

10 Xin X, Luan X, Xiao J, Wei D, Wang J, Lu D \& Yang S. Association study of four activity SNPs of CYP $3 A 4$ with the precocious puberty in Chinese girls. Neuroscience Letters 2005381 284-288.

11 Zhang LJ. Diagnosis and treatment of precocious puberty. Journal of Practical Training of Medicine 200533 5-6.

12 Oerter KE, Uriarte MM, Rose SR, Barnes KM \& Cutler GB Jr. Gonadotropin secretory dynamics during puberty in normal girls and boys. Journal of Clinical Endocrinology and Metabolism 199071 1251-1258.

13 Xiao Z, Xiao J, Jiang Y, Zhang S, Yu M, Zhao J, Wei D \& Cao H. A novel method based on ligase detection reaction for low abundant YIDD mutants detection in Hepatitis B virus. Hepatology Research 200634 150-155.

14 Stephens M, Smith NJ \& Donnelly P. A new statistical method for haplotype reconstruction from population data. American Journal of Human Genetics 200168 978-989.

15 Ohtaki T, Shintani Y \& Honda S. Metastasis suppressor gene KiSS1 encodes peptide ligand of a G-protein-coupled receptor. Nature $2001411613-617$.

16 Muir AI, Chamberlain L \& Elshourbagy NA. AXOR12, a novel human $\mathrm{G}$ proteincoupled receptor, activated by the peptide KiSS-1. Journal of Biological Chemistry 2001276 28969-28975.

17 Shahab M, Mastronardi C, Seminara SB, Crowley WF, Ojeda SR \& Plant TM. Increased hypothalamic GPR 54 signaling: a potential mechanism for initiation of puberty in primates. PNAS 2005102 2129-2134.

18 Seminara SB, Messager S, Chatzidaki EE, Thresher RR, Acierno JS, Shagoury JK, Bo-Abbas Y, Kuohung W, Schwinof KM, Hendrick AG, Zahn D, Dixon J, Kaiser UB, Slaugenhaupt SA, Gusella JF, O'Rahilly S, Carlton MBL, Crowley WF, Aparicio S \& Colledge WH. The GPR 54 gene as a regulator of puberty. New England Journal of Medicine 2003349 1614-1627.

19 de Roux N, Genin E, Carel J-C, Matsuda F, Chaussain J-L \& Milgrom E. Hypogonadotropic hypogonadism due to loss of function of the KiSS1-derived peptide receptor GPR54. PNAS $200310010972-10976$.

20 Herman-Giddens ME. Recent data on pubertal milestones in United States children: the secular trend toward earlier development. International Journal of Andrology 200629 241-246.

Received 4 February 2007

Accepted 16 April 2007 